\title{
Calcitonin gene-related peptide protects rats from cerebral ischemia/reperfusion injury via a mechanism of action in the MAPK pathway
}

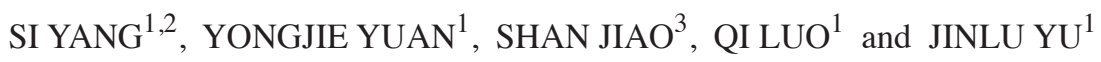 \\ Departments of ${ }^{1}$ Neurosurgery and ${ }^{2}$ Pediatric Neurology, The First Hospital of Jilin University; \\ ${ }^{3}$ Department of Endodontics, Stomatological Hospital of Jilin University, Changchun, Jilin 130021, P.R. China
}

Received January 21, 2016; Accepted April 14, 2016

DOI: $10.3892 /$ br. 2016.658

\begin{abstract}
The aim of the present study was to investigate the protective function and underlying mechanism of calcitonin gene-related peptide (CGRP) on cerebral ischemia/reperfusion damage in rats. Adult male Wistar rats were selected for the establishment of an ischemia/reperfusion injury model through the application of a middle cerebral artery occlusion. Animals were randomly divided into 6 groups of 24 animals. Drugs were administered according to the design of each group; animals were administered CGRP, CGRP8-37, PD98059 and SB20358. The neurobehavioral scores of the rat cerebral ischemia model in each group were calculated. The infarction range of the rat brain tissues was observed by 2,3,5-triphenyltetrazolium chloride staining. The expression levels of three proteins, phosphorylated c-Jun N-terminal kinase (JNK)/JNK, phosphorylated extracellular signal-regulated protein kinase (ERK)/ERK and p-p38/p38, in the mitogen-activated protein kinase (MAPK) pathway in the brain tissues was detected by western blotting. The results showed that CGRP could improve the neurobehavioral function of the ischemic rats and reduce the infarction range. Western blotting results confirmed that the function of the CGRP was mediated mainly through the reduction of the JNK and p38 phosphorylation and the promotion of ERK phosphorylation. Therefore, the present study confirmed that an increase in the exogenous CRGP could effectively improve ischemia/reperfusion injury of the brain tissue. The mechanisms of action were achieved through a reduction in JNK and p38 phosphorylation and an increase in ERL phosphorylation in the MAPK pathway. These mechanisms were interdependent.
\end{abstract}

Correspondence to: Professor Jinlu Yu, Department of Neurosurgery, The First Hospital of Jilin University, 71 Xinmin Avenue, Changchun, Jilin 130021, P.R. China

E-mail: jinluyu@hotmail.com

Key words: calcitonin gene-related peptide, cerebral ischemia, reperfusion, MAPK pathway

\section{Introduction}

During cerebral ischemia/reperfusion, the level of the calcitonin gene-related peptide (CGRP) in the neurons of the brain tissue shows a relative increase. CGRP has a role in the dilation of the cerebral blood vessels and has an important protective function in neuronal cells $(1,2)$. The exogenous supplementation of the CGRP can improve cerebral ischemia (3). However, the specific mechanism of action of the CGRP in the brain tissue remains to be elucidated. Currently, studies have been published regarding the function of the mitogen-activated protein kinase (MAPK) signal transduction pathways in cerebral ischemia/reperfusion, including the extracellular signal-regulated protein kinase (ERK), the c-Jun N-terminal kinase (JNK) and the p38 pathways $(4,5)$. However, the association between the protective effect of CGRP on brain tissues and these three signaling pathways remains to be elucidated. Therefore, the present study used cerebral ischemia/reperfusion rats as the study subjects to detect the expression levels of proteins in the aforementioned pathways and brain infarction areas to investigate the protective function of CGRP in the brain tissue of cerebral ischemia/reperfusion rats and the association of CGRP with the JNK, P38 and ERK signaling pathways.

\section{Materials and methods}

Animals and reagents. All animal manipulations were approved by the Ethics Committee of Jilin University (Changchun, China). Healthy male Wistar rats (provided by the Experimental Animal Center of Jilin University) with body weights of 250-300 g were selected. The reagents, including CGRP, the CGRPinhibitor CGRP8-37, theERK inhibitor PD98059, and the p38 inhibitor SB203580, were purchased from Sigma-Aldrich (St. Louis, MO, USA). The animals were randomly divided into 6 groups: i) The sham-surgery group (sham group), ii) the middle cerebral artery occlusion (MCAO) group, iii) the MACO-CGRP group, iv) the MCAO+CGRP+CGRP8-37 group, v) the MCAO+CGRP+PD98059 group, and vi) the $\mathrm{MCAO}+\mathrm{CGRP}+\mathrm{SB} 203580$ group. Each group comprised of 24 animals. Drugs were administered according to the design of each group. CGRP ( $3 \mathrm{~g} / \mathrm{kg})$ was intravenously injected; 
CGRP8-37 (2.5 mg/kg) was intravenously injected; PD980549 $(1 \mathrm{mg} / \mathrm{kg})$ was intraperitoneally injected; and SB203580 ( $5 \mathrm{mg} / \mathrm{kg}$ dissolved in $5 \mathrm{mg} / \mathrm{ml}$ dimethyl sulfoxide) was intraperitoneally injected.

Establishment of a cerebral ischemia/reperfusion model in rats. Before the start of the experiments, the animals were fasted for $12 \mathrm{~h}$ and water deprived for $4 \mathrm{~h}$. Following chloral hydrate anesthesia and disinfection, the carotid, internal carotid and external carotid arteries were separated. One thread of nylon monofilament line coated with poly-L-lysine was inserted along the internal carotid artery; when resistance was sensed, the monofilament was assumed to have reached the initial site of the middle cerebral artery. Muscle and skin were temporarily full-layer sutured. After $2 \mathrm{~h}$ of cerebral ischemia, the nylon line was removed and the incision was sutured. Subsequently, reperfusion was performed for $24 \mathrm{~h}$. Following surgery, the rats were placed under an illuminating lamp to maintain the body temperature of the rats between $37-37.5^{\circ} \mathrm{C}(6)$.

Neurobehavioral scoring of the rats. After the rats had awakened, the neurobehavioral function of the rats was observed during the reperfusion period. The Longa scoring standards were adopted, in which the neurological findings were scored on a 5-point scale: A score of 0 indicated no neurological deficit; a score of 1 (failure to extend left forepaw fully), a mild focal neurological deficit; a score of 2 (circling to the left), a moderate focal neurological deficit; a score of 3 (falling to the left), a severe focal deficit; and a score of 4 , inability to walk spontaneously and a depressed level of consciousness (7). Animals with scores between 1 and 3 points were selected as experimental subjects. Animals with a score of 0 or 4 points indicated the model establishment was not successful, and these animals were excluded. Animals with scores that met the above requirements but experienced a subarachnoid hemorrhage were excluded, and new animals were supplemented.

2,3,5-triphenyltetrazolium chloride (TTC) staining and measurement of the cerebral infarction areas. For the TTC staining method, subsequent to the rats being anesthetized, the brain was obtained by decapitation. The olfactory bulb and lower brain stem were separated and carefully removed. After being rapid-frozen in a $-20^{\circ} \mathrm{C}$ freezer for $\sim 20 \mathrm{~min}$, the brain tissues were sliced into five pieces with a thickness of $\sim 2 \mathrm{~mm}$. The tissues were stained in TTC buffer for $\sim 30 \mathrm{~min}$ and were fixed in $4 \%$ paraformaldehyde buffer. Normal tissues showed a pink or red color, and ischemic tissues were white (8). Images were captured using a high-resolution camera. The infarction area in the brain tissues was calculated using ImageJ software (9).

Detection of protein expression in the brain tissues by western blotting. After reperfusion for $24 \mathrm{~h}$, the brain tissues were isolated from the ischemic penumbra cortices and homogenized in ice cold buffer $[1.5 \mathrm{mmol} / \mathrm{l}$ Tris based- $\mathrm{HCl}(\mathrm{pH} 7.6)$, $1 \mathrm{mmol} / 1$ dithiothreitol, $0.25 \mathrm{~mol} / 1$ sucrose, $1 \mathrm{mmol} / 1 \mathrm{MgCl}_{2}$, $1.25 \mu \mathrm{g} / \mathrm{ml}$ pepstatin A, $10 \mu \mathrm{g} / \mathrm{ml}$ leupeptin, $2.5 \mu \mathrm{g} / \mathrm{ml}$ aprotinin, $0.5 \mathrm{mmol} / 1$ phenylmethane sulfonyl fluoride, $2.5 \mathrm{mmol} / 1$ ethylenediaminetetraacetic acid, $1 \mathrm{mmol} / \mathrm{l}$ ethylene glycol
Table I. Effects of CGRP on the neurobehavioral scores of rats with focal cerebral ischemia.

\begin{tabular}{lc}
\hline Group & Neurobehavioral scores \\
\hline Sham & $0.00 \pm 0.00$ \\
MCAO & $3.75 \pm 0.36^{\mathrm{a}}$ \\
MCAO+CGRP & $2.13 \pm 0.29^{\mathrm{a}, \mathrm{b}}$ \\
MCAO+CGRP+CGRP8-37 & $3.50 \pm 0.33^{\mathrm{a}, \mathrm{c}}$ \\
MCAO+CGRP+SB203580 & $3.25 \pm 0.31^{\mathrm{a}, \mathrm{c}}$ \\
MCAO+CGRP+PD98059 & $3.63 \pm 0.38^{\mathrm{a}, \mathrm{c}}$
\end{tabular}

${ }^{\text {aP }}<0.001$ compared to the Sham group; ${ }^{\text {b }} \mathrm{P}<0.001$ compared to the MCAO group; ${ }^{\mathrm{P}}<0.001$ compared to the $\mathrm{MCAO+CGRP}$ group. CGRP, calcitonin gene-related peptide; MCAO, middle cerebral artery occlusion.

tetraacetic acid, $0.1 \mathrm{~mol} / 1 \mathrm{Na}_{3} \mathrm{VO}_{4}, 50 \mathrm{mmol} / \mathrm{lNaF}$ and $2 \mathrm{mmol} / \mathrm{l}$ sodium pyrophosphate]. The homogenates were centrifuged $\left(1,000 \mathrm{x} \mathrm{g}\right.$ for $20 \mathrm{~min}$ at $\left.4^{\circ} \mathrm{C}\right)$ and the protein concentration of the supernatants (containing cytoplasm) and pellets (containing nucleus) was measured. Western blotting was conducted with $10 \%$ sodium dodecyl sulfate-polyacrylamide gel electrophoresis. Following electrophoresis, the proteins were transferred onto polyvinylidene fluoride membranes. The membranes were probed with the following primary antibodies: Anti-JNK (cat. no. sc-571; 1:500, polyclonal rabbit anti-rat), anti-p-JNK (cat. no. sc-135642; 1:500, polyclonal rabbit anti-rat), anti-p38 (cat. no. sc-535; 1:500, polyclonal rabbit anti-rat), anti-p-p38 (cat. no. sc-17852-R; 1:500, polyclonal rabbit anti-rat), and anti- $\beta$-actin (cat. no. sc-130657; 1:500, polyclonal rabbit anti-rat) (Santa Cruz Biotechnology, Inc., Santa Cruz, CA, USA). The horseradish peroxidase-conjugated goat anti-rabbit secondary antibody (cat. no. 7074; 1:2,000, polyclonal goat anti-rabbit) was obtained from Cell Signaling Technology (Danvers, MA, USA). Following incubation with horseradish peroxidase-conjugated goat anti-rabbit immunoglobulin G, the blots were washed, and the immunoreactive proteins were visualized on Kodak X-omat LS film (Eastman Kodak Co., New Haven, CT, USA) with enhanced chemiluminescence. The densitometry was performed with Kodak ID image analysis software (Eastman Kodak Co.).

Statistical analysis. The statistical analyses were performed using SPSS 21.0 (IBM, Corp., Armonk, NY, USA). Measurement data are represented as mean \pm standard deviation. The data were analyzed by analysis of variance, followed by SNK test. $\mathrm{P}<0.05$ was considered to indicate a statistically significant difference.

\section{Results}

Neurobehavioral scores of the rats. Compared to the MCAO group, the neurobehavioral score of the MCAO+CGRP group was low. Compared to the MCAO+CGRP group, the neurobehavioral scores of the MCAO+CGRP+CGRP8-37, $\mathrm{MCAO+CGRP+PD} 98059$ and MCAO+CGRP+SB20358 groups increased significantly (Table I). 
Table II. Effects of CGRP on the cerebral infarction area of rats with focal cerebral ischemia.

\begin{tabular}{lc}
\hline Group & Infarction area, $\%$ \\
\hline Sham & $0.0 \pm 0.0$ \\
MCAO & $40.1 \pm 2.5^{\mathrm{a}}$ \\
MCAO+CGRP & $22.7 \pm 1.3^{\mathrm{a}, \mathrm{b}}$ \\
MCAO+CGRP+CGRP8-37 & $35.2 \pm 2.8^{\mathrm{a}, \mathrm{c}}$ \\
MCAO+CGRP+SB203580 & $34.2 \pm 2.6^{\mathrm{a}, \mathrm{c}}$ \\
MCAO+CGRP+PD98059 & $35.3 \pm 3.4^{\mathrm{a}, \mathrm{c}}$ \\
\hline
\end{tabular}

${ }^{\text {aP }}<0.001$ compared to the Sham group; ${ }^{\text {b }}<0.001$ compared to the MCAO group; ${ }^{\mathrm{C}}<0.01$ compared to the MCAO+CGRP group. CGRP, calcitonin gene-related peptide; MCAO, middle cerebral artery occlusion.

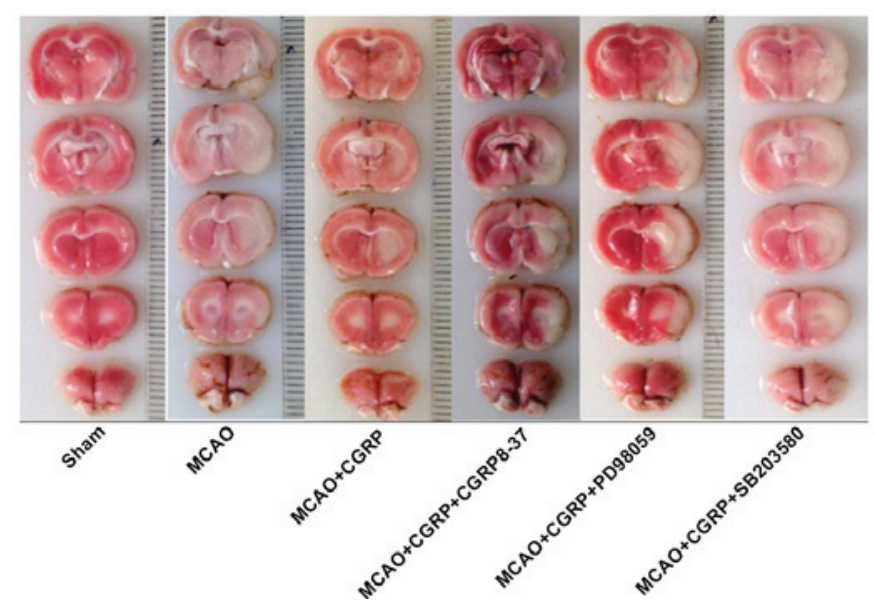

Figure 1. TTC staining in all the treatment groups. Normal tissues were a pink or red color, whereas the ischemic tissues were white. Compared to the sham group, the white area of TTC staining in the brain tissues in the MCAO group significantly increased. Compared to the MCAO group, the white area in the MCAO+CGRP group significantly decreased. Compared to the MCAO+CGRP group, the white area in the MCAO+CGRP+CGRP8-37, $\mathrm{MCAO+CGRP+PD9805}$ and MCAO+CGRP+SB203580 groups significantly increased. TTC, 2,3,5-triphenyltetrazolium chloride; CGRP, calcitonin gene-related peptide; MCAO, middle cerebral artery occlusion.

TTC staining and infarction areas in the brain tissues. Compared to the sham group, the area of white in the TTC staining of the brain tissues in the MCAO group significantly increased. Compared to the MCAO group, the area of white in the MCAO+CGRP group significantly decreased. Compared to the MCAO+CGRP group, the area of white in the 3 groups with the addition of the inhibitor groups (MCAO+CGRP+CGRP8-37, MCAO+CGRP+PD9805 and MCAO+CGRP+SB203580) significantly increased (Fig. 1 and Table II).

Western blotting results. p-JNK, JNK, p-p38, p38, p-ERK and ERK were detected. Compared to the sham group, the expression levels of p-JNK and p-p38 in the brain tissues in the MCAO group significantly increased $(\mathrm{P}<0.05)$, whereas no significant change was observed for the expression levels of JNK and p38. Compared to the MCAO group, the

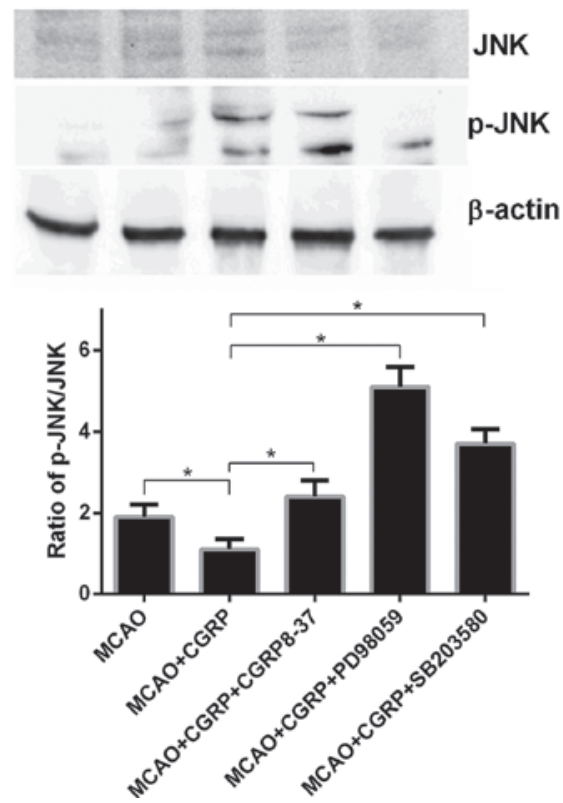

Figure 2. Western blotting results of p-JNK/JNK in all the treatment groups Compared to the MCAO group, the p-JNK expression level significantly decreased in the MCAO+CGRP group; in addition, the JNK expression level did not significantly change. Compared to the MCAO+CGRP, the p-JNK expression levels in the MCAO+CGRP+CGRP8-37,MCAO+CGRP+PD98059 and MCAO+CGRP+SB203580 groups significantly increased, whereas no change in the JNK expression level was identified. The comparison between groups was considered to indicate significant differences, "P<0.05. p-JNK, phosphorylated c-Jun N-terminal kinase; CGRP, calcitonin gene-related peptide; MCAO, middle cerebral artery occlusion.

expression levels of p-JNK and p-p38 in the MCAO+CGRP group significantly decreased $(\mathrm{P}<0.05)$; in addition, no significant changes in the expression levels of JNK and p38 were identified. Compared to the $\mathrm{MCAO}+\mathrm{CGRP}$ group, the expression levels of p-JNK and p-p38 significantly increased in the $\mathrm{MCAO}+\mathrm{CGRP}+\mathrm{CGRP} 8-37, \mathrm{MCAO}+\mathrm{CGRP}+\mathrm{PD} 98059$ and $\mathrm{MCAO}+\mathrm{CGRP}+\mathrm{SB} 203580$ groups $(\mathrm{P}<0.05)$, whereas no significant change in the protein expression levels of JNK and p38 was identified (Figs. 2 and 3).

Compared to the sham group, the expression level of p-ERK in the brain tissues in the MCAO groups significantly decreased $(\mathrm{P}<0.05)$, whereas no significant change in the ERK expression level was identified. Compared to the MCAO group, p-ERK expression in brain tissues in the MCAO+CGRP group significantly increased $(\mathrm{P}<0.05)$; whereas no significant change in ERK expression was identified. Compared to the $\mathrm{MCAO}+\mathrm{CGRP}$ group, the expression level of $\mathrm{p}-\mathrm{ERK}$ in the $\mathrm{MCAO}+\mathrm{CGRP}+\mathrm{CGRP} 8-37, \mathrm{MCAO}+\mathrm{CGRP}+\mathrm{PD} 98059$ and $\mathrm{MCAO}+\mathrm{CGRP}+\mathrm{SB} 203580$ groups significantly decreased $(\mathrm{P}<0.05)$; the reduction in the $\mathrm{MCAO}+\mathrm{CGRP}+\mathrm{PD} 98059$ group was evident; and no significant change in the ERK expression level was identified (Fig. 4).

\section{Discussion}

CGRP is a polypeptide composed of 37 amino acids, and it is extensively expressed in the central and peripheral nervous systems. Currently, CGRP is considered the most potent vasodilator $(10,11)$. CGRP serves a protective function in brain tissues. When brain tissues suffer ischemia, they are induced to 


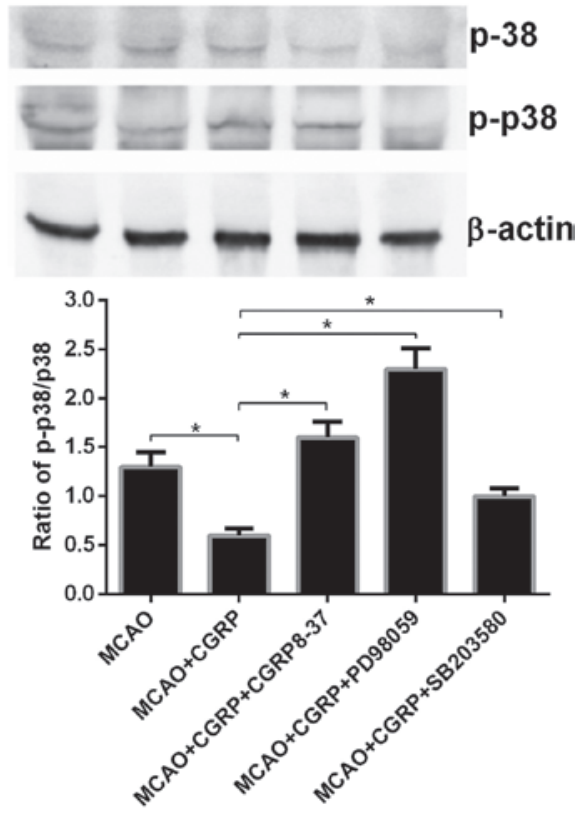

Figure 3. Western blotting results of p-p38/p38 in all the treatment groups. Compared with the MCAO group, the p-p38 expression level in the MCAO+CGRP group significantly decreased $(\mathrm{P}<0.05)$; in addition, the p38 expression level did not show a significant change. Compared to the MCAO+CGRP group, the p-p38 expression levels in the MCAO+CGRP+CGRP8-37, MCAO+CGRP+PD98059 and $\mathrm{MCAO}+\mathrm{CGRP}+\mathrm{SB} 203580$ groups significantly increased $(\mathrm{P}<0.05)$, whereas no significant change in the p38 protein expression levels was identified. The comparison between groups was considered to indicate significant differences, ${ }^{*} \mathrm{P}<0.05$. CGRP, calcitonin gene-related peptide; MCAO, middle cerebral artery occlusion.

produce numerous self-protective substances; of these, CGRP is one of the most important (3). Studies have shown that during cerebral ischemia/reperfusion, the CGRP level in the neuronal cells in the brain tissue exhibits a relative increase. This increase is intended to cause dilation of the cerebral blood vessels to provide blood supply to the ischemic brain tissue as quickly as possible. However, when the cerebral ischemia was relatively severe, the CGRP produced by the brain tissues could not completely defend this injury; therefore, severe cerebral ischemia occurred (12). Thus, the concept that an increase in exogenous CGRP could relieve ischemia/reperfusion brain injury was a possibility. The results from the present study show that, in an ischemia/reperfusion rat model, the intravenous injection of exogenous CGRP can effectively improve the neurobehavioral function of rats and increase their quality of life. Pathological examination of the brain tissue showed that CGRP effectively decreased the infarction area in the rat brain tissue induced by the suture. These results could be blocked by the CGRP inhibitor CGRP8-37. Although CGRP could effectively relieve nerve injury caused by ischemia/reperfusion, the specific mechanism of the CGRP remains to be elucidated.

Studies have shown that in the cerebral ischemia/reperfusion injury model, the MAPK signal transduction pathways have important roles in cell apoptosis (13-15). The MAPK signal transduction pathways mainly include the ERK, JNK and $\mathrm{p} 38$ pathways. The functions of these three signaling pathways in cerebral ischemia/reperfusion are different (16-18). Studies have shown that the JNK signaling pathway has an important role in the programmed cell death process following

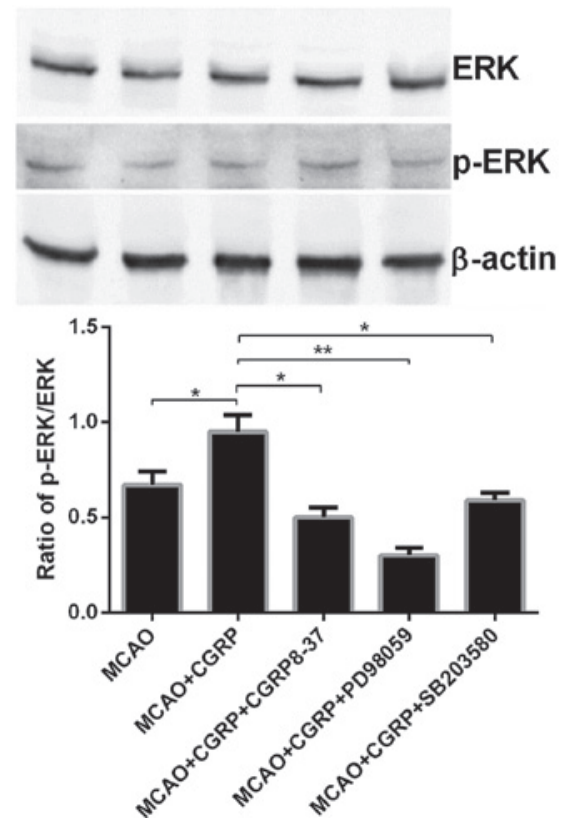

Figure 4. Western blotting results of p-ERK/ERK in all the treatment groups. Compared to the MCAO group, the p-ERK expression level significantly increased in the brain tissues in the MCAO+CGRP group; whereas no significant change in the ERK expression was identified. Compared to the MCAO+CGRP group, the expression level of the p-ERK significantly decreased in the MCAO+CGRP+CGRP8-37, MCAO+CGRP+PD98059 and

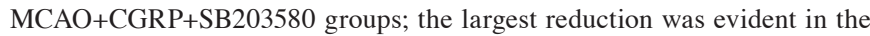
$\mathrm{MCAO+CGRP+PD98059}$ group, whereas no significant change in the expression level of the ERK protein was observed. The comparison between groups was considered to indicate significant differences, ${ }^{*} \mathrm{P}<0.05$ and ${ }^{* * *} \mathrm{P}<0.01$. CGRP, calcitonin gene-related peptide; MCAO, middle cerebral artery occlusion.

cerebral ischemia/reperfusion injury and can promote cell apoptosis (19). The p38 signaling pathway is activated at the early stage of cerebral ischemia to allow participation in free radical injury, cell apoptosis, cytotoxicity, and the inflammatory reaction (20). The p38 inhibitor has an important protective function in neurons (16). The classic ERK signaling pathway has important roles in numerous diseases such as myocardial infarction, tumor and diabetes mellitus (21-23). Following cerebral ischemia/reperfusion, the ERK signaling pathway was activated to reduce the NMDA receptor activity in cerebral ischemia/reperfusion injury, inhibit the intracellular $\mathrm{Ca} 2+$ influx, and has an important protective role in neuronal function (4). These pathways also should experience similar changes in the cerebral ischemia/reperfusion models in rats. However, the mechanism underlying the protection of brain tissues by CGRP and the association between this protective function and the three signaling pathways of the MAPK family remain to be elucidated. The present study exogenously supplemented CGRP and used the CGRP inhibitor CGRP8-37, the ERK inhibitor PD98059 and the p38 inhibitor SB203580 to investigate this association.

The results of the study showed that in the cerebral ischemia/reperfusion model in rats, an exogenous increase in the CGRP significantly increased the phosphorylation level of ERK as shown by western blotting. In addition, TTC staining showed that the infarction area significantly decreased. When the ERK inhibitor PD98059 and CGRP were administered together, the western blotting results showed that the phosphorylation level 
of ERK significantly decreased. In addition, TTC staining showed that the infarction area in the brain tissues significantly increased. These results indicate that an exogenous increase in CGRP could exert a protective function in nerve cells; however, following the inhibition of the ERK signaling pathway, the protective function of CGRP was no longer evident. Therefore, the CGRP function may be achieved through the activation of the ERK pathway and the promotion of its phosphorylation. Analysis of the JNK and p38 signaling pathways showed that following the administration of CGRP, the phosphorylation levels of the JNK and p38 significantly decreased according to western blotting. In addition, the TTC staining results showed that the infarction area significantly decreased. The p38 inhibitor SB203580 could reverse the protective function of CGRP; thus, the p38 inhibitor could influence the phosphorylation of JNK and p38 simultaneously. Therefore, the present study demonstrated that in the cerebral ischemia/reperfusion model, the JNK and p38 signaling pathways were significantly activated, whereas the ERK signaling pathway experienced certain inhibitions. When the CGRP level in the brain tissues was exogenously increased, the expression levels of p-JNK and p-p38 decreased, and the protein expression level of p-ERK increased. In addition, CGRP could significantly improve the neuronal function in the brain tissues and decrease the occurrence of cell apoptosis. Therefore, CGRP may achieve the protective function on neurons in the brain tissues via its influence on the JNK, p38 and ERK signaling pathways. With the addition of the inhibitors of these three MAPK pathways, the protective function of the exogenously increased CGRP attenuated or disappeared, indicating that these three pathways are interdependent. The protective function of CGRP could not be achieved when any one of these pathways was inhibited.

\section{Acknowledgements}

The present study was supported by the National Natural Science Foundation of China (grant no. 81200888).

\section{References}

1. Zhang ZH, Fang XB, Xi GM, Li WC, Ling HY and Qu P: Calcitonin gene-related peptide enhances CREB phosphorylation and attenuates tau protein phosphorylation in rat brain during focal cerebral ischemia/reperfusion. Biomed Pharmacother 64: 430-436, 2010.

2. Liu Z,Liu Q, Cai H, Xu C, Liu G and Li Z: Calcitonin gene-related peptide prevents blood-brain barrier injury and brain edema induced by focal cerebral ischemia reperfusion. Regul Pept 171: 19-25, 2011.

3. Shao B, Zhou YL, Wang H and Lin YS: The role of calcitonin gene-related peptide in post-stroke depression in chronic mild stress-treated ischemic rats. Physiol Behav 139: 224-230, 2015.

4. Zhou J, Du T, Li B, Rong Y, Verkhratsky A and Peng L: Crosstalk between MAPK/ERK and PI3K/AKT signal pathways during brain ischemia/reperfusion. ASN Neuro 7: 7, 2015.

5. Wei SG, Yu Y, Weiss RM and Felder RB: Inhibition of brain mitogen-activated protein kinase signaling reduces central endoplasmic reticulum stress and inflammation and sympathetic nerve activity in heart failure rats. Hypertension 67: 229-236, 2016.
6. Nagasawa H and Kogure K: Correlation between cerebral blood flow and histologic changes in a new rat model of middle cerebral artery occlusion. Stroke 20: 1037-1043, 1989.

7. Longa EZ, Weinstein PR, Carlson S and Cummins R: Reversible middle cerebral artery occlusion without craniectomy in rats. Stroke 20: 84-91, 1989.

8. Bederson JB, Pitts LH, Germano SM, Nishimura MC, Davis RL and Bartkowski HM: Evaluation of 2,3,5-triphenyltetrazolium chloride as a stain for detection and quantification of experimental cerebral infarction in rats. Stroke 17: 1304-1308, 1986.

9. Schneider CA, Rasband WS and Eliceiri KW: NIH Image to Image J: 25 years of image analysis. Nat Methods 9: 671-675, 2012.

10. Mistrova E, Wiegand S, Sviglerova J, Pfeil U, Kuncova J, Slavikova J, Kummer W and Chottova Dvorakova M: Adrenomedullin and the calcitonin receptor-like receptor system mRNA expressions in the rat heart and sensory ganglia in experimentally-induced long-term diabetes. Gen Physiol Biophys 33: 215-225, 2014.

11. Hagner S, Stahl U, Knoblauch B, McGregor GP and Lang RE: Calcitonin receptor-like receptor: Identification and distribution in human peripheral tissues. Cell Tissue Res 310: 41-50, 2002.

12. Cai H, Xu X, Liu Z, Wang Q, Feng G, Li Y, Xu C, Liu G and $\mathrm{Li}$ Z: The effects of calcitonin gene-related peptide on bFGF and AQP4 expression after focal cerebral ischemia reperfusion in rats. Pharmazie 65: 274-278, 2010.

13. Zhang CX, Liu JX, Li D, Li L, Fu JH, Hou JC, Du XM and Zhang FC: Effect of guanmaitong tablet on ERK and $\mathrm{p} 38$ protein of TLR2 pathway expression in cerebral ischemia/reperfusion rats: An experimental study. Zhongguo Zhong Xi Yi Jie He Za Zhi 35: 712-716, 2015 (In Chinese).

14. Nozaki K, Nishimura M and Hashimoto N: Mitogen-activated protein kinases and cerebral ischemia. Mol Neurobiol 23: 1-19, 2001.

15. Sun X, Liu C, Qian M, Zhao Z and Guo J: Ceramide from sphingomyelin hydrolysis differentially mediates mitogen-activated protein kinases (MAPKs) activation following cerebral ischemia in rat hippocampal CA1 subregion. J Biomed Res 24: 132-137, 2010.

16. Piao CS, Kim JB, Han PL and Lee JK: Administration of the p38 MAPK inhibitor SB203580 affords brain protection with a wide therapeutic window against focal ischemic insult. J Neurosci Res 73: 537-544, 2003.

17. Bonny C, Borsello T and Zine A: Targeting the JNK pathway as a therapeutic protective strategy for nervous system diseases. Rev Neurosci 16: 57-67, 2005.

18. Haddad JJ: The role of Bax/Bcl-2 and pro-caspase peptides in hypoxia/reperfusion-dependent regulation of MAPK(ERK): Discordant proteomic effect of MAPK(p38). Protein Pept Lett 14: 361-371, 2007.

19. Shen CP, Tsimberg Y, Salvadore C and Meller E: Activation of Erk and JNK MAPK pathways by acute swim stress in rat brain regions. BMC Neurosci 5: 36, 2004.

20. Piao CS, Che Y, Han PL and Lee JK: Delayed and differential induction of p38 MAPK isoforms in microglia and astrocytes in the brain after transient global ischemia. Brain Res Mol Brain Res 107: 137-144, 2002.

21. Jiang M, Wang L and Jiang HH: Role of spinal MAPK-ERK signal pathway in myocardial ischemia-reperfusion injury. Zhongguo Dang Dai Er Ke Za Zhi 15: 387-391, 2013 (In Chinese).

22. Anfuso CD, Motta C, Giurdanella G, Arena V, Alberghina M and Lupo G: Endothelial PKC $\alpha$-MAPK/ERK-phospholipase A2 pathway activation as a response of glioma in a triple culture model. A new role for pericytes? Biochimie 99: 77-87, 2014.

23. Kim TK, Lee JS, Jung HS, Ha TK, Kim SM, Han N, Lee EJ, Kim TN, Kwon MJ, Lee SH, et al: Triiodothyronine induces proliferation of pancreatic $\beta$-cells through the MAPK/ERK pathway. Exp Clin Endocrinol Diabetes 122: 240-245, 2014. 